Pacific Journal of Mathematics

JENSEN POLYNOMIALS AND THE TURÁN AND LAGUERRE
INEQUALITIES AND GEORGE LESLIE CSORDAS 


\section{JENSEN POLYNOMIALS AND THE TURÁN AND LAGUERRE INEQUALITIES}

\section{Thomas Craven and George Csordas}

In this paper we will (1) establish a relationship between the Turán inequalities and the Laguerre inequalities, (2) provide a complete characterization of functions in the Laguerre-Pólya class in terms of the Turán inequalities involving the Jensen polynomials and (3) show that certain Hankel determinants of functions in the LaguerrePólya class are nonpositive.

Introduction. A real entire function $\psi(x)$ is said to be in the LaguerrePólya class if $\psi(x)$ can be represented in the form

$$
\psi(x):=c x^{m} e^{-\alpha x^{2}+\beta x} \prod_{k=1}^{\infty}\left(1+x / x_{k}\right) e^{-x / x_{k}},
$$

where $c, \beta, x_{k}$ are real, $\alpha \geq 0, m$ is a nonnegative integer and $\sum x_{k}^{-2}<\infty$. Pólya and Schur [PS] termed an entire function $\varphi(x)$ as a function of type I in the Laguerre-Pólya class if $\varphi(x)$ or $\varphi(-x)$ can be represented in the form

$$
\varphi(x):=c x^{m} e^{\sigma x} \prod_{k=1}^{\infty}\left(1+x / x_{k}\right),
$$

where $\sigma \geq 0, c$ is real, $m$ is a nonnegative integer, $x_{k}>0$, and $\sum x_{k}^{-1}<$ $\infty$. If $\varphi(x)$ is in the Laguerre-Pólya class, we will write $\varphi \in \mathscr{L}-\mathscr{P}$ or $\varphi \in \mathscr{L}-\mathscr{P}(\mathrm{II})$. Also, if $\varphi(x)$ is a function of type I in $\mathscr{L}-\mathscr{P}$, we will write $\varphi(x) \in \mathscr{L}-\mathscr{P}(\mathrm{I})$. Now it is clear that if $\varphi \in \mathscr{L}-\mathscr{P}(\mathrm{I})$, then $\varphi \in \mathscr{L}$ $\mathscr{P}($ II).

For the various properties of functions in the Laguerre-Pólya class we refer the reader to [PS], [O], [CC] and the references contained therein. For the reader's convenience, we single out here a few known facts [PS] concerning functions in the Laguerre-Pólya class. For a function

$$
\varphi(x):=\sum_{k=0}^{\infty} \frac{\gamma_{k}}{k !} x^{k}
$$

in the Laguerre-Pólya class the following statements are valid. 
(i) For each $n$, the Jensen polynomials

$$
g_{n}(x):=\sum_{k=0}^{n}\left(\begin{array}{l}
n \\
k
\end{array}\right) \gamma_{k} x^{k}, \quad n=0,1,2, \ldots,
$$

have only real zeros.

(ii) For each $k$, the Turán inequalities hold; that is,

$$
T_{k}:=\gamma_{k}^{2}-\gamma_{k-1} \gamma_{k+1} \geq 0, \quad k=1,2,3, \ldots
$$

(iii) For each $p$, the Laguerre inequalities hold [S]; that is,

$$
\left(\varphi^{(p)}(x)\right)^{2}-\varphi^{(p-1)}(x) \varphi^{(p+1)}(x) \geq 0,
$$

for all real $x$ and $p=1,2,3, \ldots$

(iv) If $\gamma_{k} \geq 0$ for $k=0,1,2, \ldots$, then $\varphi(x) \in \mathscr{L}-\mathscr{P}(\mathbf{I})$.

In $\S 2$, after reviewing some of the known properties of Jensen polynomials, we shall relate the Turán inequalities for an arbitrary entire function to the Laguerre inequalities and to Turán-type inequalities of the Jensen polynomials on the positive real axis (Theorem 2.3). We then prove that the requirement that the inequalities hold on the whole real axis forces $\varphi(x)$ to be in $\mathscr{L}-\mathscr{P}$. This culminates in a complete characterization of $\mathscr{L}-\mathscr{P}(\mathrm{II})$ in Theorem 2.7. The section ends with a proof of a related problem of Karlin.

In $\S 3$, we shall cite two open problems and give several examples which illustrate the necessity of the hypotheses in our results.

2. The main results. We begin this section with the terminology and notation we will use in the sequel. If

$$
f(x):=\sum_{k=0}^{\infty} \frac{\gamma_{k}}{k !} x^{k}
$$

is a real entire function, so that $\gamma_{k} \in \mathbf{R}, k=0,1,2, \ldots$, then the $n$th Jensen polynomial associated with $f(x)$ is defined by

$$
g_{n}(t):=\sum_{k=0}^{n}\left(\begin{array}{l}
n \\
k
\end{array}\right) \gamma_{k} t^{k} \quad(n=0,1,2, \ldots) .
$$

The $n$th Jensen polynomial associated with $f^{(p)}(x), p=0,1,2, \ldots$, will be denoted by

$$
g_{n, p}(t):=\sum_{k=0}^{n}\left(\begin{array}{l}
n \\
k
\end{array}\right) \gamma_{k+p} t^{k} \quad(n, p=0,1,2, \ldots) .
$$


The $n$th Appell polynomial associated with $f(x)$, if $\gamma_{0} \neq 0$, is defined by

$$
P_{n}(t):=\frac{1}{n !} \sum_{k=0}^{n}\left(\begin{array}{l}
n \\
k
\end{array}\right) \gamma_{k} t^{n-k} \quad(n=0,1,2, \ldots)
$$

If

$$
h(x):=\sum_{k=0}^{n} a_{k} x^{k}, \quad a_{n} \neq 0,
$$

is any polynomial, we define

$$
h^{*}(x):=x^{n} h\left(x^{-1}\right), \quad(x \neq 0)
$$

In particular if $\gamma_{n} \neq 0$, then with the above definition, (2.3) and (2.4) we have

$$
\begin{cases}g_{n}^{*}(t):=t^{n} g_{n}\left(t^{-1}\right) & (n=0,1,2, \ldots, t \neq 0), \\ P_{n}(t)=\left(\frac{1}{n !}\right) g_{n}^{*}(t) & (n=0,1,2, \ldots) .\end{cases}
$$

Some of the properties of the polynomials are summarized in the following proposition (see, for example, Csordas and Varga [CV]).

Proposition 2.1. The following properties hold for the functions defined by (2.1)-(2.5).

(i) If $\gamma_{0} \neq 0, P_{n}^{\prime}(t)=P_{n-1}(t)(t \in \mathbf{R}, n=1,2,3, \ldots)$.

(ii) The sequence $\left\{g_{n}(t)\right\}_{n=0}^{\infty}$ is generated by $e^{x} f(x t)$; that is,

$$
e^{x} f(x t)=\sum_{n=0}^{\infty} g_{n}(t) \frac{x^{n}}{n !} \quad(x, t \in \mathbf{R}) .
$$

If $\gamma_{0} \neq 0$, the sequence $\left\{n ! P_{n}(t)\right\}_{n=0}^{\infty}$ is generated by $e^{x t} f(x)$; that is,

$$
e^{x t} f(x)=\sum_{n=0}^{\infty} P_{n}(t) x^{n}=\sum_{n=0}^{\infty} g_{n}^{*}(t) \frac{x^{n}}{n !} \quad(x, t \in \mathbf{R})
$$

$$
n g_{n}(t)=n g_{n-1}(t)+t g_{n}^{\prime}(t) \quad(t \in \mathbf{R}, n=1,2,3, \ldots)
$$

$$
g_{n+1, p}(t)=g_{n, p}(t)+t g_{n, p+1}(t) \quad(t \in \mathbf{R}, n, p=0,1,2, \ldots) .
$$

(v) If

$$
\begin{array}{r}
\Delta_{n, p}(t):=g_{n, p}^{2}(t)-g_{n-1, p}(t) g_{n+1, p}(t) \\
\quad(n=1,2,3, \ldots, p=0,1,2, \ldots),
\end{array}
$$


then

$$
\begin{aligned}
\Delta_{n, p}(t)=t^{2}\left[g_{n-1, p+1}^{2}(t)-\right. & \left.g_{n-1, p}(t) g_{n-1, p+2}(t)\right], \\
& (n=1,2,3, \ldots, p=0,1,2, \ldots) .
\end{aligned}
$$

Proof. Direct verification yields (i)-(iv) (for (ii) and (iii), see for example, Rainville [R, p. 133]). To prove (v), we use (2.9) in the form

$$
g_{n, p}(t)=g_{n-1, p}(t)+t_{n-1, p+1}(t) .
$$

Substituting (2.9) and (2.12) into (2.10) yields (v).

The Jensen polynomials associated with an arbitrary entire function form a "natural" sequence of approximating polynomials. This property will be needed below and is established in the following lemma.

LEMMA 2.2. Let

$$
h(z):=\sum_{k=0}^{\infty} \frac{a_{k}}{k !} z^{k} \quad\left(a_{k} \in \mathbf{C}\right),
$$

be an arbitrary entire function. For each fixed nonnegative integer $p$, let

$$
g_{n, p}(z):=\sum_{k=0}^{n}\left(\begin{array}{l}
n \\
k
\end{array}\right) a_{k+p} z^{k} \quad(n=0,1,2, \ldots)
$$

Then

$$
\lim _{n \rightarrow \infty} g_{n, p}\left(\frac{z}{n}\right)=h^{(p)}(z) \quad(p=0,1,2, \ldots)
$$

uniformly on compact subsets of $\mathbf{C}$.

Proof. For a fixed nonnegative integer $p$ and for $n \geq 2$, we have

$$
\begin{aligned}
g_{n, p}\left(\frac{z}{n}\right)= & a_{p}+a_{p+1} z \\
& +\sum_{k=2}^{n}\left(1-\frac{1}{n}\right) \cdots\left(1-\frac{k-1}{n}\right) \frac{a_{k+p}}{k !} z^{k} .
\end{aligned}
$$

Then, by the Cauchy inequalities for the Taylor coefficients of $h^{(p)}(z)$, we have

$$
\left|\frac{a_{k+p}}{k !}\right| \leq \frac{M\left(R, h^{(p)}\right)}{R^{k}} \quad(k=1,2,3, \ldots, R>0) .
$$


where

$$
M\left(R, h^{(p)}\right)=\max _{|z|=R}\left|h^{(p)}(z)\right| .
$$

Thus, for $n>m+1$ and for $0<|z| \leq r<R=2 r$, we obtain

$$
\begin{gathered}
\left|\sum_{k=m+1}^{n}\left(1-\frac{1}{n}\right) \cdots\left(1-\frac{k-1}{n}\right) \frac{a_{k+p}}{k !} z^{k}\right| \\
\leq M\left(R, h^{(p)}\right) \frac{r^{m+1}}{R^{m}(R-r)},
\end{gathered}
$$

and

$$
\left|\sum_{k=m+1}^{\infty} \frac{a_{k+p}}{k !} z^{k}\right| \leq M\left(R, h^{(p)}\right) \frac{r^{m+1}}{R^{m}(R-r)} .
$$

Therefore, with $R=2 r$ and for any $\varepsilon>0$, there is a positive integer $m_{0}$ such that $r^{m+1}\left(R^{m}(R-r)\right)^{-1}<\varepsilon$ for all $m \geq m_{0}$. Finally, there is a positive integer $N>m_{0}$ such that for all $n \geq N$ and $|z| \leq r$

$$
\left|\sum_{k=2}^{m_{0}}\left(1-\frac{1}{n}\right) \cdots\left(1-\frac{k-1}{n}\right) \frac{a_{k+p}}{k !} z^{k}-\sum_{k=2}^{m_{0}} \frac{a_{k+p}}{k !} z^{k}\right|<\varepsilon,
$$

and hence (2.15) follows from (2.19), (2.20) and (2.21).

With the aid of the foregoing preliminary results we will now prove

THEOREM 2.3. Suppose

$$
f(x):=\sum_{k=0}^{\infty} \frac{\gamma_{k}}{k !} x^{k}, \quad \gamma_{k}>0, k=0,1,2, \ldots,
$$

is an entire function. Then the following are equivalent.

$$
T_{k}:=\gamma_{k}^{2}-\gamma_{k-1} \gamma_{k+1} \geq 0 \quad \text { for } k=1,2,3, \ldots
$$

$$
\Delta_{n, p}(t):=g_{n, p}^{2}(t)-g_{n-1, p}(t) g_{n+1, p}(t) \geq 0,
$$

for all $t \geq 0$ and $n=1,2,3, \ldots, p=0,1,2, \ldots$.

$$
L_{p+1}(f(t)):=\left(f^{(p+1)}(t)\right)^{2}-f^{(p)}(t) f^{(p+2)}(t) \geq 0
$$

for all $t \geq 0$ and $p=0,1,2, \ldots$.

Proof. (2.23) $\Rightarrow$ (2.24). Assuming (2.23) (with $\left.\gamma_{k}>0, k=0,1,2, \ldots\right)$ we will establish (2.24) by induction. First, we set $t=1$. Then, for 
$n=1$, we have by $(2.23)$

(2.26)

$$
\begin{aligned}
& g_{1, p}^{2}(1)-g_{0, p}(1) g_{2, p}(1) \\
& =\gamma_{p+1}^{2}-\gamma_{p} \gamma_{p+2} \geq 0, \quad p=0,1,2, \ldots
\end{aligned}
$$

Next, we assume that

$$
\Delta_{k, p}(1) \geq 0 \text { for } k=1, \ldots, n \text { and } p=0,1,2, \ldots
$$

Then by the induction assumption (2.27) and by Proposition 2.1(v) (cf. (2.11)) we have

$$
\begin{aligned}
\Delta_{n, p}(1)=g_{n-1, p+1}^{2}(1)-g_{n-1, p}(1) g_{n-1, p+2}(1) & \geq 0, \\
p & =0,1,2, \ldots,
\end{aligned}
$$

and

(2.29)

$$
\begin{aligned}
\frac{g_{n-1, p+1}(1)}{g_{n-1, p}(1)} & \geq \frac{g_{n-1, p+2}(1)}{g_{n-1, p+1}(1)} \\
& \geq \frac{g_{n-1, p+3}(1)}{g_{n-1, p+2}(1)}, \quad p=0,1,2, \ldots .
\end{aligned}
$$

Let $p$ be a fixed, but arbitrary, nonnegative integer. Then by (2.11),

$$
\Delta_{n+1, p}(1)=g_{n, p+1}^{2}(1)-g_{n, p}(1) g_{n, p+2}(1),
$$

and by (2.9),

$$
g_{n, p}(1)=g_{n-1, p}(1)+g_{n-1, p+1}(1) .
$$

Thus, if we apply (2.31) to (2.30), then after some simplifications, (2.30) becomes

$$
\text { (2.32) } \begin{aligned}
\Delta_{n+1, p}(1)= & \Delta_{n, p}(1)+\Delta_{n, p+1}(1)+g_{n-1, p+1}(1) g_{n-1, p+2}(1) \\
& -g_{n-1, p}(1) g_{n-1, p+3}(1)
\end{aligned}
$$

Hence, it follows from (2.28) and (2.29) that $\Delta_{n+1, p}(1) \geq 0$. Since $p$ was an arbitrary nonnegative integer we conclude that $\Delta_{n+1, p}(1) \geq 0$ for $n, p=0,1,2, \ldots$.

Now if $t_{0} \geq 0, \alpha_{k} \geq 0, k=0,1,2, \ldots$, and if $\alpha_{k}^{2}-\alpha_{n-1} \alpha_{n+1} \geq 0$, then with $\gamma_{k}:=t_{0}^{k} \alpha_{k}, k=0,1,2, \ldots$, it follows that

$$
\gamma_{k}^{2}-\gamma_{k-1} \gamma_{k+1}=t_{0}^{2 k}\left(\alpha_{k}^{2}-\alpha_{k-1} \alpha_{k+1}\right) \geq 0, \quad k=1,2,3, \ldots
$$

In light of these considerations, we see that (2.24) holds for all $t \geq 0$. $(2.24) \Rightarrow(2.25)$. Suppose (2.24) holds. Then by (2.11) and (2.24),

$$
0 \leq \Delta_{n, p}(t)=t^{2}\left[g_{n-1, p+1}^{2}(t)-g_{n-1, p}(t) g_{n-1, p+2}(t)\right]
$$


for all $t \geq 0, n=1,2,3, \ldots, p=0,1,2, \ldots$. Therefore, by Lemma 2.2 (cf. $(2.15))$ we see that

$$
\begin{aligned}
\lim _{n \rightarrow \infty} & {\left[g_{n-1, p+1}^{2}\left(\frac{t}{n-1}\right)\right.} \\
& \left.-g_{n-1, p}\left(\frac{t}{n-1}\right) g_{n-1, p+2}\left(\frac{t}{n-1}\right)\right] \\
& =\left[f^{(p+1)}(t)\right]^{2}-f^{(p)}(t) f^{(p+2)}(t) \geq 0,
\end{aligned}
$$

for all $t \geq 0$ and for all $p=0,1,2, \ldots$.

Since the implication $(2.25) \Rightarrow(2.23)$ is immediate, the proof of the theorem is complete.

REMARK 2.4. Inequalities (2.23) and (2.24) are called the Turán inequalities. A comprehensive treatment of these inequalities and their applications in the theory of orthogonal polynomials may be found in Karlin and Szegö [KS] and the references contained therein. The inequalities (2.25) are frequently termed the Laguerre inequalities (see, for example, Skovgaard [S] or Patrick [P]). The Laguerre inequalities play an important role in the theory of the distribution of zeros of several classes of entire functions. See, for example, the recent proof of the Pólya-Wiman conjecture, Craven, Csordas and Smith [CCS], where these inequalities were used in the following, perhaps more familiar form,

$$
\frac{d^{2}}{d t^{2}}\left[\log f^{(p)}(t)\right] \leq 0
$$

Thus by (2.35) (and under the equivalent conditions of Theorem 2.3), $f^{(p)}(t)$ is logarithmically concave for $t \geq 0$, where $f(t)$ is defined by (2.22). Therefore, it follows that $\log (1 / f(t))$ is convex for $t \geq 0$. In particular, Theorem 2.3 also generalizes a result of Vincze [V]. In his study of the characterization of the Poisson and gamma distributions, Vincze shows, in particular, that if $\gamma_{k}>0$ and

$$
\gamma_{k}^{2}-\frac{k}{k+1} \gamma_{k-1} \gamma_{k+1}>0, \quad k=1,2,3, \ldots,
$$

then

$$
\frac{d^{2}}{d t^{2}}\left[\frac{1}{f^{(p)}(t)}\right]=\frac{2\left[f^{(p+1)}(t)\right]^{2}-f^{(p)}(t) f^{(p+2)}(t)}{\left[f^{(p)}(t)\right]^{3}}>0
$$

for $t>0, p=0,1,2, \ldots$. (Note that the open problem of Rényi and Vincze, discussed in [V], has been recently solved by Miles and 
Williamson [MW].) But because of the factor of " 2 " in (2.36), this inequality does not imply the Laguerre inequalities (2.25). On the other hand, we were able to extend Vincze's ingenious argument to deduce (2.25) directly from (2.23). However, this argument is somewhat involved and since it does not yield (2.24) we will omit it here. Finally, it is evident that Theorem 2.3 remains valid, mutatis mutandis, if we merely assume that the function $f(x)$ in $(2.22)$ is a real analytic function with positive Taylor coefficients.

Next we consider the case when (2.23) is replaced by the following requirements

$$
\left\{\begin{array}{l}
\Delta_{n}(t):=\Delta_{n, 0}(t)>0 \text { for all } t \neq 0, n=0,1,2, \ldots \\
\text { and } \\
\gamma_{k+1}^{2}-\gamma_{k} \gamma_{k+2}>0, \gamma_{k}>0 \text { for } k=0,1,2, \ldots
\end{array}\right.
$$

Then (2.37) constitutes a very strong set of conditions (see, for example, Csordas and Williamson [CW1] and [CW2]). Indeed, if (2.37) holds, then all the Jensen polynomials associated with $f(x)$ of $(2.22)$ have only simple real zeros. Therefore, the following theorem is of particular interest.

THEOREM 2.5. Let

$$
\varphi(x):=\sum_{k=0}^{\infty} \frac{\gamma_{k}}{k !} x^{k}, \quad \gamma_{k} \neq 0,
$$

denote a real entire function and let $g_{n}(x), n=0,1,2, \ldots$, denote the nth Jensen polynomial associated with $\varphi(x)$. Then the following conditions are equivalent for any $n \geq 1$.

(i) $\Delta_{n}(t)>0$ for all real $t \neq 0$ and $\gamma_{n}^{2}-\gamma_{n-1} \gamma_{n+1}>0$.

(ii) $\Delta_{n}^{*}(t)>0$ for all real $t$.

(iii) For each $k=1,2,3, \ldots, n$, and for real $t_{0}$, if $g_{k}\left(t_{0}\right)=0$, then $g_{k-1}\left(t_{0}\right) g_{k+1}\left(t_{0}\right)<0$, and if $\gamma_{n}=0$, then $\gamma_{n-1} \gamma_{n+1}<0$.

(iv) For each $k=1,2,3, \ldots, n$, and for real $t_{0}$, if $g_{k}^{*}\left(t_{0}\right)=0$, then $g_{k-1}^{*}\left(t_{0}\right) g_{k+1}^{*}\left(t_{0}\right)<0$.

(v) $g_{n+1}(t)$ has only simple real zeros.

Proof. For each fixed $n$, we will prove the following implications: (ii) $\Leftrightarrow($ i $) \Rightarrow($ iii $) \Leftrightarrow($ iv $) \Rightarrow(v) \Rightarrow($ ii). Note that $(\mathrm{i}) \Rightarrow($ iii) is trivial. Since $\Delta_{n}^{*}(t)=t^{2 n} \Delta_{n}\left(t^{-1}\right)$ for $t \neq 0$, and $\Delta_{n}^{*}(0)=\gamma_{n}^{2}-\gamma_{n-1} \gamma_{n+1}$, it is clear that (i) $\Leftrightarrow\left(\right.$ ii). Similarly, using the fact that $g_{n}^{*}(t)=t^{n} g_{n}\left(t^{-1}\right)$ for $t \neq$ 0 , and $g_{n}^{*}(0)=\gamma_{n}$, we see that (iii) $\Leftrightarrow(\mathrm{iv})$. Next, assume that (iv) 
holds. Since $\gamma_{0} \neq 0$ by assumption (cf. (2.38)), and $(d / d t) g_{n+1}^{*}(t)=$ $(n+1) g_{n}^{*}(t)$ and since (iv) holds, the sequence $g_{n+1}^{*}(t), g_{n}^{*}(t), \ldots, g_{0}^{*}(t)$ $=\gamma_{0}$, is a Sturm sequence for the interval $(-\infty, \infty)$ (see, for example, Jacobson, [J, pp. 278-283] or Wilf [W, pp. 90-95]). Therefore by Sturm's theorem [J, p. 283], the number of distinct real zeros of $g_{n+1}^{*}$ in $(-\infty, \infty)$ is equal to the number of sign changes in the sequence $\left\{(-1)^{n+1} \gamma_{0},(-1)^{n} \gamma_{0}, \ldots, \gamma_{0}\right\}$, minus the number of changes of sign in the sequence $\left\{\gamma_{0}, \gamma_{0}, \ldots, \gamma_{0}\right\}$ of leading coefficients. Since this number is $n+1$, we see that $g_{n+1}^{*}(t)$, and hence $g_{n+1}(t)$, have only simple real zeros. This proves that (iv) $\Rightarrow(\mathrm{v})$.

Finally, suppose that (v) holds. Since by definition (cf. (2.2)), $\operatorname{deg} g_{n+1}^{*} \leq n+1$ and by (v) $g_{n+1}^{*}$ has $(n+1)$ distinct zeros, $\operatorname{deg} g_{n+1}^{*}=$ $n+1$. Let

$$
g_{n+1}^{*}(t):=\gamma_{0} \prod_{j=1}^{n+1}\left(t-\alpha_{j}\right) \quad\left(\alpha_{1}<\alpha_{2}<\cdots<\alpha_{n+1}\right)
$$

Then for all real $t, t \neq \alpha_{j}, j=1, \ldots, n+1$,

$$
\begin{aligned}
\frac{d^{2}}{d t^{2}} \log g_{n+1}^{*}(t) & =\frac{d}{d t} \frac{(n+1) g_{n}^{*}(t)}{g_{n+1}^{*}(t)}=\frac{d}{d t} \sum_{j=1}^{n+1} \frac{1}{\left(t-\alpha_{j}\right)} \\
& =-\sum_{j=1}^{n+1} \frac{1}{\left(t-\alpha_{j}\right)^{2}}<0 .
\end{aligned}
$$

Since the zeros of $g_{n+1}^{*}(t)$ are all simple, the Cauchy-Schwarz inequality yields

$$
\left(\sum_{j=1}^{n+1} \frac{1}{t-\alpha_{j}}\right)^{2}<(n+1) \sum_{j=1}^{n+1} \frac{1}{\left(t-\alpha_{j}\right)^{2}}
$$

Thus, we have by (2.40), for real $t \neq \alpha_{j}, j=1, \ldots, n+1$,

$$
\begin{gathered}
\left(g_{n+1}^{* \prime}(t)\right)^{2}-g_{n+1}^{*}(t) g_{n+1}^{* \prime \prime}(t)=\left(g_{n+1}^{*}(t)\right)^{2} \sum_{j=1}^{n+1} \frac{1}{\left(t-\alpha_{j}\right)^{2}} \\
>\frac{\left(g_{n+1}^{*}(t)\right)^{2}}{n+1}\left(\sum_{j=1}^{n+1} \frac{1}{\left(t-\alpha_{j}\right)}\right)^{2}=\frac{\left(g_{n+1}^{* \prime}(t)\right)^{2}}{n+1}
\end{gathered}
$$


Therefore,

$$
\begin{aligned}
0 & <\left(1-\frac{1}{n+1}\right)\left(g_{n+1}^{* \prime}(t)\right)^{2}-g_{n+1}^{*}(t) g_{n+1}^{* \prime \prime}(t) \\
& =\frac{n}{n+1}(n+1)^{2}\left(g_{n}^{*}(t)\right)^{2}-(n+1) \cdot n g_{n+1}^{*}(t) g_{n-1}^{*}(t) \\
& =n(n+1) \Delta_{n}^{*}(t),
\end{aligned}
$$

and so we see that $\Delta_{n}(t)>0$ for all $t \neq 0$ and $n=1,2,3, \ldots$ This completes the proof of the theorem.

Next we recall that by a classical result of Pólya and Schur [PS], a real entire function $\varphi(x) \in \mathscr{L}-\mathscr{P}$ if and only if the associated Jensen polynomials $g_{n}(x), n=1,2,3, \ldots$, have only real zeros. Hence, the following corollary is an immediate consequence of Theorem 2.15.

COROLLARY 2.6. Let $\varphi(x)$ be the real entire function defined by (2.38). If the equivalent conditions (i)-(v) of Theorem 2.5 hold for $n=1,2,3, \ldots$, then $\varphi(x) \in \mathscr{L}-\mathscr{P}(\mathrm{II})$.

To obtain a complete characterization of the functions in $\mathscr{L}-\mathscr{P}(\mathrm{II})$, we must allow the possibility that $\Delta_{n}(t)$ equals zero in Theorem 2.5, or equivalently, that the polynomial $g_{n}(t)$ has multiple zeros. This is done in the next theorem.

TheOREM 2.7. Let $\varphi(x)=\sum_{k=0}^{\infty} \gamma_{k} x^{k} / k$ ! denote a real entire function and let $g_{n}(t), n=0,1,2, \ldots$ and $\Delta_{n}(t), n=1,2,3, \ldots$ be defined by (2.2) and (2.37) respectively. Then $\varphi(x) \in \mathscr{L}-\mathscr{P}(\mathrm{II})$ if and only if the following conditions hold:

(i) $\Delta_{n}(t) \geq 0$ for all real $t$ and $n=1,2,3, \ldots$

(ii) If $\gamma_{0} \neq 0$ and $\gamma_{1}^{2}-\gamma_{0} \gamma_{2}>0$, then (a) $g_{n+1}\left(t_{0}\right)=0$ whenever $\Delta_{n}\left(t_{0}\right)=0, t_{0} \neq 0$, and (b) $\gamma_{n+1}=0$ whenever $\gamma_{n}^{2}-\gamma_{n-1} \gamma_{n+1}=0$.

(iii) If $\gamma_{0} \neq 0$ and $\gamma_{1}^{2}-\gamma_{0} \gamma_{2}=0$, then $\varphi(x)=\gamma_{0} e^{\gamma_{1} x / \gamma_{0}}$.

(iv) If $\gamma_{0}=0$, then $\varphi(x)=x^{r} \psi(x)$ with $\psi(0) \neq 0$ where $\psi$ satisfies (i), (ii) and (iii) for the appropriately redefined $\gamma_{n}, g_{n}$ and $\Delta_{n}$.

Note that condition (ii)(b) is essentially the case $t_{0}=\infty$ in condition (ii)(a). We shall begin our proof of this theorem with two lemmas.

LEMMA 2.8. With $\varphi(x), \Delta_{n}$ and $g_{n}$ as in the theorem, assume that $\Delta_{n}(t) \geq 0$ for all real $t$ and $n=1,2,3, \ldots$. If $\Delta_{n}\left(t_{0}\right)=0, t_{0} \neq 0$, then $g_{n+1}\left(t_{0}\right) \Delta_{n-1}\left(t_{0}\right)=0$. 
Proof. Set $u=t_{0}^{-1}$. Then we have

$$
\Delta_{n}^{*}(u)=0=\left(g_{n}^{*}(u)\right)^{2}-g_{n-1}^{*}(u) g_{n+1}^{*}(u),
$$

and $u$ must be a multiple zero of $\Delta_{n}^{*}$ since $\Delta_{n}^{*} \geq 0$. Therefore, using (2.5) and Proposition 2.1(i),

$$
0=\Delta_{n}^{* \prime}(u)=(n-1)\left[g_{n}^{*}(u) g_{n-1}^{*}(u)-g_{n+1}^{*}(u) g_{n-2}^{*}(u)\right] .
$$

If $g_{n}^{*}(u) \neq 0$, we can use (2.42) to deduce that the right hand side of (2.43) equals

$$
\begin{aligned}
& \frac{n-1}{g_{n}^{*}(u)}\left[g_{n}^{* 2}(u) g_{n-1}^{*}(u)-g_{n}^{*}(u) g_{n+1}^{*}(u) g_{n-2}^{*}(u)\right] \\
& \quad=\frac{n-1}{g_{n}^{*}(u)} g_{n+1}^{*}(u) \Delta_{n-1}^{*}(u),
\end{aligned}
$$

and thus $g_{n+1}\left(t_{0}\right) \Delta_{n-1}\left(t_{0}\right)=0$. On the other hand, if $g_{n}^{*}(u)=0$, then (2.43) implies either $g_{n+1}^{*}(u)=0$ or, if $g_{n+1}^{*}(u) \neq 0$, then $g_{n-2}^{*}(u)=0$ and $g_{n-1}^{*}(u)=0$ (cf. $\left.(2.42)\right)$ so that $\Delta_{n-1}^{*}(u)=0$. Again we have $g_{n+1}\left(t_{0}\right) \Delta_{n-1}\left(t_{0}\right)=0$.

LEMMA 2.9. Under the hypotheses of Theorem 2.7, assume that $\Delta_{n}(t)$ $\geq 0$ for all real $t$ and $n=1,2,3, \ldots$, and that $\gamma_{1}^{2}-\gamma_{0} \gamma_{2} \neq 0$. If for some $n, \Delta_{n}\left(t_{0}\right)=0, t_{0} \neq 0$ and $g_{n+1}\left(t_{0}\right)=0$, then $g_{n+1}$ has at least two nonreal zeros.

Proof. By Lemma 2.8, we have $\Delta_{n-1}\left(t_{0}\right)=0$. By hypothesis, $\Delta_{1}\left(t_{0}\right)$ $=\left(\gamma_{1}^{2}-\gamma_{0} \gamma_{2}\right) t_{0}^{2} \neq 0$. If we let $k$ be the largest integer less than $n$ with $\Delta_{k}\left(t_{0}\right) \neq 0$, then Lemma 2.8 , together with a simple induction gives $g_{k+1}\left(t_{0}\right)=\cdots=g_{n}\left(t_{0}\right)=0$. Writing $u=t_{0}^{-1}$, we have $g_{n+1}^{* \prime}(u)=$ $(n+1) g_{n}^{*}(u)=0$ and $g_{n+1}^{* \prime \prime}(u)=n(n+1) g_{n-1}^{*}(u)=0$. Since $g_{n+1}^{*}(u) \neq 0$, an elementary geometric argument shows that $g_{n+1}^{*}$, and hence $g_{n+1}$, have nonreal zeros.

Proof of Theorem 2.7. We first consider necessity. Assume that $\varphi(x) \in \mathscr{L}-\mathscr{P}(\mathrm{II})$. Then, for any fixed $t$, we have $e^{x} \varphi(x t) \in \mathscr{L}-\mathscr{P}(\mathrm{II})$, so that (i) is just the Turán inequalities for the coefficients in the series in (2.6). Condition (ii) holds by Lemma 2.8 and 2.9. Condition (iii) was proved in [CC, Proposition 4.5]. When $\varphi(x)=x^{r} \psi(x)$ with $\psi(0) \neq 0$, then $\psi(x)=\sum_{k=0}^{\infty} \alpha_{k} x^{k} / k$ ! with $\alpha_{k}=\gamma_{k+r} /(k+r) \cdots(k+1)$ and

$$
g_{n}(x)=n(n-1) \cdots(n-r+1) x^{r} \sum_{k=0}^{n-2}\left(\begin{array}{c}
n-r \\
k
\end{array}\right) \alpha_{k} x^{k}
$$


Since $\psi(x) \in \mathscr{L}-\mathscr{P}(\mathrm{II})$, the above argument shows that (i)-(iii) must hold for $\psi(x)$.

Conversely, assume conditions (i)-(iv) hold. By (iv), we may assume $\gamma_{0}=\varphi(0) \neq 0$. Fix $n>0$. As noted previously, Pólya and Schur [PS] have shown that it will suffice to show that $g_{n}$ (or equivalently, $g_{n}^{*}$ ) has only real zeros. Consider the sequence of polynomials

$$
g_{0}^{*}, g_{1}^{*}, \ldots, g_{n}^{*}
$$

If $\Delta_{k}^{*}(t)>0$ for all $t, k<n$, then this sequence is a Sturm sequence for $g_{n}^{*}$ as in the proof of Theorem 2.5. And, as in that proof, the number of real zeros of $g_{n}^{*}$ is $n$. Again by Theorem 2.5, this holds whenever we have strict inequality in (i) for $t \neq 0$ and $\gamma_{k}^{2}-\gamma_{k-1} \gamma_{k+1}>0$, $k=1, \ldots, n-1$. Next suppose that $\Delta_{n}^{*}(t) \geq 0$ for all real $t$. By (iii), we are done if $\gamma_{1}^{2}-\gamma_{0} \gamma_{2}=0$, so we may assume $\gamma_{1}^{2}-\gamma_{0} \gamma_{2}>0$ and thus the hypotheses of (ii) hold. Now let $u$ be such that $\Delta_{k}^{*}(u)=0$ for some $k \leq n$, where $k$ is minimal for $u$. Note that there are only finitely many such points $u$ since $\Delta_{k} \equiv 0$ implies $g_{k+1} \equiv 0$ by (ii)(a), which in turn implies $\gamma_{0}=0$, contrary to assumption. From (ii) and $\Delta_{k}^{*}(u)=0$, we obtain $g_{k}^{*}(u)=g_{k+1}^{*}(u)=0$, so that $\Delta_{k+1}^{*}(u)=0$. (If $u=0$, then $\Delta_{k}^{*}(u)=\gamma_{k}^{2}-\gamma_{k-1} \gamma_{k+1}=0$ and (ii)(b) is used.)

An induction using (ii) gives us $g_{k}^{*}(u)=g_{k+1}^{*}(u)=\cdots=g_{n}^{*}(u)=0$, with $g_{k-1}^{*}(u) \neq 0$ (by the minimality of $k$ ). Since $(d / d x) g_{j}^{*}(x)=$ $j g_{j-1}^{*}(x)$ for each $j$, we have a sequence of polynomials

$$
g_{0}^{*}(x), \ldots, g_{k-1}^{*}(x), g_{k}^{*}(x) /(x-u), \ldots, g_{n}^{*}(x) /(x-u)^{n-k+1},
$$

which are all nonzero at $x=u$. It follows that (2.45) forms a Sturm sequence for $g_{n}^{*}(x) /(x-u)^{n-k+1}$ for any interval containing $u$ and excluding other multiple zeros of $g_{n}^{*}$ : indeed, for $x$ near $u$, but $x \neq u$, $g_{j}^{*}(x)=0$ implies that $g_{j-1}^{*}(x) /(x-u)^{l}$ and $g_{j+1}^{*}(x) /(x-u)^{l+2}$ have opposite signs since $(x-u)^{l}(x-u)^{l+2}>0$ and $g_{j-1}^{*}(x) g_{j+1}^{*}(x)<0$ by (i). Eliminating each of the multiple zeros of $g_{n}^{*}$ in this way gives a Sturm sequence, where the leading coefficient of all the polynomials is still $\gamma_{0}$ as in (2.44). It follows that the resulting quotient of $g_{n}^{*}$ has only real zeros, and hence so does $g_{n}^{*}$.

We next consider an open problem of Karlin [K, pp. 389-390]. Let

$$
\varphi(x):=\sum_{k=0}^{\infty} \frac{\gamma_{k}}{k !} x^{k}
$$


be a real entire function and let $H_{3}\left(\varphi^{(p)} ; x\right), p=0,1,2, \ldots$, denote the $3 \times 3$ Hankel determinant associated with $\varphi^{(p)}(x)$ :

$$
H_{3}\left(\varphi^{(p)} ; x\right):=\operatorname{det}\left[\begin{array}{ccc}
\varphi^{(p)}(x) & \varphi^{(p+1)}(x) & \varphi^{(p+2)}(x) \\
\varphi^{(p+1)}(x) & \varphi^{(p+2)}(x) & \varphi^{(p+3)}(x) \\
\varphi^{(p+2)}(x) & \varphi^{(p+3)}(x) & \varphi^{(p+4)}(x)
\end{array}\right]
$$

We will prove below that if $\varphi(x) \in \mathscr{L}-\mathscr{P}$ and $\gamma_{k}>0, k=0,1,2, \ldots$, then $H_{3}\left(\varphi^{(p)} ; x\right) \leq 0$ for all $x \geq 0$. To this end we will need the following lemma.

LEMMA 2.10. (i) If $f(x):=e^{\sigma x} q(x)$, where $\sigma \in \mathbf{R}$ and $q(x) \in \mathbf{R}[x]$, then

$$
H_{3}(f ; x)=e^{3 \sigma x} H_{3}(q ; x), \quad x \in \mathbf{R} .
$$

(ii) Let $q(x) \in \mathscr{L}-\mathscr{P}$ and suppose that $q(x)$ has nonnegative Taylor coefficients. Let

$$
\begin{aligned}
G(q ; x):= & q^{2}(x) q^{\prime \prime \prime}(x)-3 q(x) q^{\prime}(x) q^{\prime \prime}(x) \\
& +2\left(q^{\prime}(x)\right)^{3}, \quad x \in \mathbf{R} .
\end{aligned}
$$

Then

$$
G(q ; x) \geq 0 \text { for } x \geq 0
$$

Proof. Part (i) follows directly from a computation. To prove (ii), we induct on the degree of $q(x)$. Clearly (2.50) holds if $\operatorname{deg} q(x)=1$. Set

$$
Q(x):=(x+a) q(x),
$$

where $a \geq 0$, and assume that (2.50) holds. Then a calculation shows that

$$
G(Q ; x)=(x+a)^{3} G(q ; x)+2(q(x))^{3} .
$$

Since $a \geq 0$ and $q(x) \geq 0$, it follows from the induction assumption that $G(Q ; x) \geq 0$ for all $x \geq 0$.

In the proof of our next theorem we will use the following generalization of the Turán and Laguerre inequalities (cf. (2.23) and (2.25)) due to Jensen [Je]. For a detailed proof of the following theorem see [CV]. 
THEOREM 2.11. With (2.46), set

$$
L_{n}(\varphi(x)):=\sum_{k=0}^{2 n} \frac{(-1)^{k+n}}{(2 n) !}\left(\begin{array}{c}
2 n \\
k
\end{array}\right) \varphi^{(k)}(x) \varphi^{(2 n-k)}(x)
$$

$$
(x \in \mathbf{R}, n=0,1,2, \ldots) \text {. }
$$

Then $\varphi(x) \in \mathscr{L}-\mathscr{P}$ if and only if

$$
L_{n}(\varphi(x)) \geq 0 \quad(x \in \mathbf{R}, n=0,1,2, \ldots) .
$$

THEOREM 2.12. Let $q(x) \in \mathbf{R}[x]$ with positive leading coefficient and suppose that the zeros of $q(x)$ are all nonpositive. Then

$$
H_{3}(q ; x) \leq 0 \text { for all } x \geq 0 \text {. }
$$

Proof. We will prove the theorem by induction on the degree of $q(x)$. Clearly, if $\operatorname{deg} q \leq 1$, then (2.55) holds, since in this case $H_{3}(q ; x) \equiv 0$. Now set

$$
Q(x):=(x+a) q(x),
$$

where $a \geq 0$ and $q(x)$ has only real nonpositive zeros. Next, we assume that $H_{3}(q ; x) \leq 0$ for all $x \geq 0$. For $x \in \mathbf{R}$, set

$$
I(Q ; x):=-Q(x) Q^{(4)}(x)+4 Q^{\prime}(x) Q^{\prime \prime \prime}(x)-3\left(Q^{\prime \prime}(x)\right)^{2} .
$$

Then, using (2.53), a computation shows that

$$
I(Q ; x)=-12 L_{2}(Q(x)) \text {. }
$$

Since $q, Q \in \mathscr{L}-\mathscr{P}$, we conclude, by Theorem 2.11 , that

$$
I(q ; x) \leq 0 \text { and } I(Q ; x) \leq 0 \text { for all } x \in \mathbf{R} .
$$

Now another computation yields that

$$
\begin{aligned}
H_{3}(Q ; x)= & H_{3}(q ; x) a^{3}+3 x H_{3}(q ; x) a^{2} \\
& +\left(3 x^{2} H_{3}(q ; x)+q(x) I(q ; x)\right) a \\
& +x^{3} H_{3}(q ; x)+x q(x) I(q ; x)-4 G(q ; x) .
\end{aligned}
$$

Now by Lemma 2.10 (cf. (2.50)), $-G(q ; x) \leq 0$ for $x \geq 0$ and by (2.59), $I(q ; x) \leq 0$ for $x \geq 0$. Thus, using the induction assumption and the fact that $a \geq 0$, we conclude that $H_{3}(Q ; x) \leq 0$ for $x \geq 0$.

We note that the use of Jensen's inequality (2.54) in the proof above could have been avoided by doing yet another induction to show that

$$
L_{2}((x+a) q(x))=(x+a)^{2} L_{2}(q(x))+\left(\left(q^{\prime}(x)\right)^{2}-q(x) q^{\prime \prime}(x)\right)
$$

is always nonnegative. 
THEOREM 2.13. Let

$$
\varphi(x):=\sum_{k=1}^{\omega} \frac{\gamma_{k}}{k !} x^{k}, \quad \gamma_{k} \geq 0,0 \leq \omega \leq \infty,
$$

be a function in the Laguerre-Polya class. Then

$$
H_{3}\left(\varphi^{(p)} ; x\right) \leq 0 \text { for all } x \geq 0, \quad p=0,1,2, \ldots,
$$

where $H_{3}\left(\varphi^{(p)} ; x\right)$ is defined by (2.47). In particular,

$$
\operatorname{det}\left[\begin{array}{ccc}
\gamma_{p} & \gamma_{p+1} & \gamma_{p+2} \\
\gamma_{p+1} & \gamma_{p+2} & \gamma_{p+3} \\
\gamma_{p+2} & \gamma_{p+3} & \gamma_{p+4}
\end{array}\right] \leq 0 \quad(p=0,1,2, \ldots) .
$$

Proof. Since $\varphi(x) \in \mathscr{L}-\mathscr{P}$, and $\gamma_{k} \geq 0, \varphi(x)$ can be expressed in the form

$$
\varphi(x)=c e^{\sigma x} \prod_{1}^{\omega}\left(1+x / x_{n}\right), \quad 0 \leq \omega \leq \infty,
$$

where $c>0, \sigma \geq 0, x_{n}>0$ and $\sum^{\omega} x_{n}^{-1}<\infty$. First, we consider the case when $p=0$. If $\omega=N<\infty$, set

$$
\varphi_{N}(x):=c e^{\sigma x} \prod_{n=1}^{N}\left(1+x / x_{n}\right) .
$$

Then by Lemma 2.10 (cf. (2.48)) and Theorem 2.12

$$
H_{3}\left(\varphi_{N} ; x\right) \leq 0 \text { for all } x \geq 0 \text {. }
$$

If $\omega=\infty$, we know that $\varphi_{N}(x) \rightarrow \varphi(x)$ as $N \rightarrow \infty$, uniformly on compact subsets of $\mathrm{C}$. Thus, it follows that $H_{3}\left(\varphi_{N} ; x\right) \rightarrow H_{3}(\varphi ; x)$ as $N \rightarrow \infty$, for $x \geq 0$. Consequently, (2.62) holds with $p=0$. Since $\mathscr{L}-\mathscr{P}$ is closed under differentiation [PS] and since $\gamma_{k} \geq 0$, it follows that for any $p=0,1,2, \ldots, \varphi^{(p)}(x)$ can also be expressed in the form (2.64), and thus (2.62) holds for $p=0,1,2, \ldots$. Since the assertion (2.63) follows from (2.62) by setting $x=0$, the proof of the theorem is complete.

Corollary 2.14. Let

$$
\varphi(x):=\sum_{k=1}^{\omega} \frac{\gamma_{k}}{k !} x^{k}, \quad \gamma_{k} \geq 0,0 \leq \omega \leq \infty,
$$


be a function in the Laguerre-Pólya class. Let

$$
T_{k}:=\gamma_{k}^{2}-\gamma_{k-1} \gamma_{k+1}, \quad k=1,2,3, \ldots
$$

Then the following extended Turán inequalities hold:

$$
E_{k}:=T_{k}^{2}-T_{k-1} T_{k+1} \geq 0, \quad k=2,3,4, \ldots
$$

Proof. A computation shows that

(2.70) $\operatorname{det}\left[\begin{array}{ccc}\gamma_{k} & \gamma_{k+1} & \gamma_{k+2} \\ \gamma_{k+1} & \gamma_{k+2} & \gamma_{k+3} \\ \gamma_{k+2} & \gamma_{k+3} & \gamma_{k+4}\end{array}\right]\left(-\gamma_{k+2}\right)=E_{k+2}, \quad k=0,1,2, \ldots$

Since $\left(-\gamma_{k+2}\right) \leq 0,(2.69)$ follows from Theorem 2.13 (cf. (2.63)).

REMARKs 2.15. (a) The inequality (2.69) provides a new, easily tested necessary condition for an entire function, with nonnegative Taylor coefficients, to have only real zeros.

(b) Let

$$
\varphi(x):=\sum_{k=0}^{\infty} \frac{\gamma_{k}}{k !} x^{k}, \quad \gamma_{k}>0,
$$

be an entire function and let $g_{n, p}^{*}(x)$ denote the $n$th Appell polynomial (cf. (2.3) and (2.5)) associated with $\varphi^{(p)}(x), p=0,1,2, \ldots$ Then a computation shows that

$$
\begin{aligned}
A_{p}(t) & :=\operatorname{det}\left[\begin{array}{lll}
g_{0, p}^{*}(t) & g_{1, p}^{*}(t) & g_{2, p}^{*}(t) \\
g_{1, p}^{*}(t) & g_{2, p}^{*}(t) & g_{3, p}^{*}(t) \\
g_{2, p}^{*}(t) & g_{3, p}^{*}(t) & g_{4, p}^{*}(t)
\end{array}\right] \\
& =\operatorname{det}\left[\begin{array}{ccc}
\gamma_{p} & \gamma_{p+1} & \gamma_{p+2} \\
\gamma_{p+1} & \gamma_{p+2} & \gamma_{p+3} \\
\gamma_{p+2} & \gamma_{p+3} & \gamma_{p+4}
\end{array}\right] .
\end{aligned}
$$

Hence, if $\varphi(x) \in \mathscr{L}-\mathscr{P}, \gamma_{k} \geq 0$, then $A_{p}(t) \leq 0$ for all $t \in \mathbf{R}$ and for $p=0,1,2, \ldots$.

3. Examples and open problems. The purpose of this section is to provide some examples which show that in a sense our results are best possible. In addition, we will cite here two open problems.

EXAMPLE 3.1. In reference to Theorem 2.3, consider

$$
f_{1}(x):=e^{3 x}-x-\frac{2}{3}=\frac{1}{3}+2 x+\sum_{k=2}^{\infty} \frac{3^{k}}{k !} x^{k} \text {. }
$$


Thus, the Turán inequalities (2.23) all hold. But then, using the notation in (2.25), we find that

$$
L_{1}\left(f_{1}(t)\right)=9 t e^{3 t}+1
$$

Since $L_{1}\left(f_{1}(-1 / 3)\right)=1-3 e^{-1}<0$, we see that, if $(2.23)$ is valid, then, in general, the Laguerre inequalities (2.25) need not hold for all real $t$.

In addition, we note that if

$$
f_{2}(x):=x+e^{x}=1+2 x+\sum_{k=2}^{\infty} \frac{x^{k}}{k !}:=\sum_{k=0}^{\infty} \frac{\gamma_{k}}{k !} x^{k},
$$

then $\gamma_{2}^{2}-\gamma_{1} \gamma_{3}=-1<0$, and $\gamma_{k}^{2}-\gamma_{k-1} \gamma_{k+1} \geq 0$ for all $k \geq 1, k \neq 2$, so that exactly one of the inequalities (2.23) fails. In this case, with the notation in (2.25), $L_{2}\left(f_{2}(t)\right)=-e^{t}<0$ for all real $t$.

EXAMPLE 3.2. In reference to Corollary 2.6 and Theorem 2.7, we now show that if

$$
f_{3}(x):=\sum_{k=0}^{\infty} \frac{\gamma_{k}}{k !} x^{k}, \quad \gamma_{0} \neq 0,
$$

is a real entire function and if

$$
\begin{aligned}
& \Delta_{n}(t)=\left(g_{n}(t)\right)^{2}-g_{n-1}(t) g_{n+1}(t) \geq 0, \\
& \quad \text { for all real } t \text { and } n=1,2,3, \ldots,
\end{aligned}
$$

where $g_{n}(t)$ is the $n$th Jensen polynomial associated with $f_{3}(x)$, then $f_{3}(x)$ need not belong to the Laguerre-Pólya class. Indeed, with $\gamma_{0}:=$ $1, \gamma_{2}:=\gamma_{3}:=0, \gamma_{4}:=-1, \gamma_{k}:=0, k=5,6,7, \ldots$, we have that $f_{3}(x)=1-x^{4} / 4$ !, $g_{n}(t)=1,0 \leq n \leq 3$ and $g_{n}(t)=1-\left(\begin{array}{l}n \\ 4\end{array}\right) t^{4}$ for $n=4,5,6, \ldots$ Then a calculation shows that $(3.5)$ is satisfied since

$$
\Delta_{n}(t):=\frac{(n-1)(n-2)}{144} t^{4}\left[(n-3)(n-2)(n-1) n t^{4}+72\right] \geq 0
$$

for all $t \geq 0, n=1,2,3, \ldots$, but $f_{3}(x)=1-x^{4} / 4$ ! is not a function in the Laguerre-Pólya class.

EXAMPLE 3.3. In reference to Theorem 2.13, consider the Hankel determinant $H_{3}(\varphi ; x)$ (see (2.47) for the notation), where $\varphi(x):=$ $(x+1)(x+2)(x+3)$. Thus, $\varphi(x) \in \mathscr{L}-\mathscr{P}(\mathrm{I})$, but a calculation shows that

$$
H_{3}(\varphi ; x)=-36(x+2)\left(x^{2}+4 x+5\right) \text {. }
$$


Consequently, $H_{3}(\varphi ; x)>0$ for $x<-2$, and $H_{3}(\varphi ; x) \leq 0$ for $x \geq-2$; that is, in general, $H_{3}(\varphi ; x)$ cannot have an invariant sign as $x$ ranges over the real axis. (This example also shows that there is a misprint in [K, p. 389, inequality (12.5) and p. 390, line 3].)

The results of Theorem 2.13 and Example 3.3 suggest the following open problem.

Open Problem 3.4 (Karlin [K]). Let $\varphi(x) \in \mathscr{L}-\mathscr{P}(\mathrm{I})$ and suppose $\varphi(x)$ has nonnegative Taylor coefficients. Let $H_{n}\left(\varphi^{(p)} ; x\right)$ denote the $n \times n$ Hankel determinant

$$
H_{n}\left(\varphi^{(p)} ; x\right):=\operatorname{det}\left[\begin{array}{cccc}
\varphi^{(p)}(x) & \varphi^{(p+1)}(x) & \cdots & \varphi^{(p+n-1)}(x) \\
\varphi^{(p+1)}(x) & \varphi^{(p+2)}(x) & \cdots & \varphi^{(p+n)}(x) \\
\vdots & \vdots & & \vdots \\
\varphi^{(p+n-1)}(x) & \varphi^{(p+n)}(x) & \cdots & \varphi^{(p+2 n-2)}(x)
\end{array}\right]
$$

where $p=0,1,2, \ldots$ Then the conjecture is that

$$
\begin{aligned}
(3.7)(-1)^{n(n-1) / 2} H_{n}\left(\varphi^{(p)} ; x\right) \geq 0 \quad \text { for } x \geq 0, & p=0,1,2,3, \ldots, \\
& \text { and for } n=4,5,6, \ldots
\end{aligned}
$$

Open Problem 3.5. In reference to Corollary 2.14 consider the function $\varphi(x)$ in $\mathscr{L}-\mathscr{P}$ defined by (2.67). Then the Turán expressions (cf. $(2.68))$

$$
T_{k}:=\gamma_{k}^{2}-\gamma_{k+1} \gamma_{k-1}, \quad k=1,2,3, \ldots,
$$

are all nonnegative. But then elementary considerations show that the function

$$
f(x):=\sum_{k=0}^{\infty} \frac{T_{k+1}}{k !} x^{k},
$$

where $T_{k}$ is defined by (3.8) is also an entire function. The open problem then is to characterize those functions $\varphi(x)$ in $\mathscr{L}-\mathscr{P}$ for which the associated functions $f(x)$ (cf. (3.9)) are also in $\mathscr{L}-\mathscr{P}$ (see also the example below).

EXAMPLE 3.6. Let

$$
\varphi_{1}(x):=\left(x^{2}+10 x+1\right) e^{x},
$$

so that $\varphi_{1}(x)$ is in $\mathscr{L}-\mathscr{P}(\mathrm{I})$. Then a calculation shows that $T_{k}=78+$ $18 k+2 k^{2}$ and that the associated entire function

$$
f_{1}(x):=\sum_{k=0}^{\infty} \frac{T_{k+1}}{k !} x^{k}=\left(98+24 x+2 x^{2}\right) e^{x} .
$$

Therefore, we see that $f_{1}(x)$ is not in $\mathscr{L}-\mathscr{P}$. 
On the other hand, if

$$
\varphi_{2}(x):=\left(x^{2}+2 x+1 / 2\right) e^{x}
$$

then $T_{k}=2 k^{2}+2 k-1$, and the associated function is

$$
f_{2}(x):=\sum_{k=0}^{\infty} \frac{T_{k+1}}{k !} x^{k}=\left(2 x^{2}+8 x+3\right) e^{x}
$$

is clearly in $\mathscr{L}-\mathscr{P}(\mathbf{I})$.

Other choices of $\varphi(x)$ for which $f(x)$, defined by (3.9), is again in $\mathscr{L}-\mathscr{P}(\mathrm{I})$ are $(x+1)^{n}$ for any positive integer $n$ and the function $\sum_{k=0}^{\infty} x^{k} /(k !)^{2}$.

\section{REFERENCES}

[CC] T. Craven and G. Csordas, Location of zeros. Part I: Real polynomials and entire functions, Illinois J. Math., 27 (1983), 244-278.

[CCS] T. Craven, G. Csordas and W. Smith, The zeros of derivatives of entire functions and the Pólya-Wiman conjecture, Ann. Math., 125 (1987), 405-431.

[CV] G. Csordas and R. S. Varga, Necessary and sufficient conditions and the Riemann hypothesis, to appear.

[CW1] G. Csordas and J. Williamson, On polynomials satisfying a Turán type inequality, Proc. Amer. Math. Soc., 43 (1974), 367-372.

[CW2] _ The zeros of Jensen polynomials are simple, Proc. Amer. Math. Soc., 49 (1975), 263-264.

[J] N. Jacobson, Lectures in Abstract Algebra, vol. III, D. van Nostrand Co., New York, 1964.

[Je] J. L. W. V. Jensen, Recherches sur la théorie des équations, Acta Math., 36 (1913), 181-195.

[K] S. Karlin, Total Positivity, vol. 1, Stanford University Press, Stanford, Calif., 1968.

[KS] S. Karlin and G. Szegö, On certain determinants whose elements are orthogonal polynomials, J. Analyse Math., 8 (1960), 1-157.

[MW] J. Miles and J. Williamson, A characterization of the exponential function, J. London Math. Soc., (2) 33 (1986), 110-116.

[O] N. Obreschkoff, Verteilung und Berechnung der Nullstellen Reeller Polynome, VEB Deutscher Verlag der Wissenschaften, Berlin, 1963.

[Pa] M. L. Patrick, Extensions of inequalities of the Laguerre and Turán type, Pacific J. Math., 44 (1973), 675-682.

[PS] G. Pólya and J. Schur, Über zwei Arten von Faktorenfolgen in der Theorie der algebraischen Gleichungen, J. Reine Angew. Math., 144 (1914), 89-113.

[R] E. D. Rainville, Special Functions, Chelsea Publishing Co., New York, 1960.

[S] H. Skovgaard, On inequalities of the Turán type, Math. Scand., 2 (1954), 67-73.

[V] I. Vincze, Contribution to a characterization problem, Proc. of the 3rd Pannonian Symp. on Math. Stat., Visegrad, Hungary (1982), 353-361. 
[W] H. S. Wilf, Mathematics for the Physical Sciences, John Wiley and Sons Inc., New York, 1962.

Received October 12, 1987.

UNIVERSITY OF HAWAII AT MANOA

HoNOLULU, HI 96822 


\section{PACIFIC JOURNAL OF MATHEMATICS \\ EDITORS}

\author{
V. S. VARADARAJAN \\ (Managing Editor) \\ University of California \\ Los Angeles, CA 90024 \\ HERBERT ClEMENS \\ University of Utah \\ Salt Lake City, UT 84112 \\ THOMAS ENRIGHT \\ University of California, San Diego \\ La Jolla, CA 92093
}

R. FINN

Stanford University

Stanford, CA 94305

HERMANN FLASCHKA

University of Arizona

Tucson, AZ 85721

VAUGhan F. R. JONES

University of California

Berkeley, CA 94720

STEVEN KERCKHOFF

Stanford University

Stanford, CA 94305

\section{ROBION KIRBY}

University of California

Berkeley, CA 94720

C. C. MOORE

University of California

Berkeley, CA 94720

HAROLD STARK

University of California, San Diego

La Jolla, CA 92093

\section{ASSOCIATE EDITORS}
R. ARENS
E. F. BECKENBACH
B. H. NEUMANN
F. WOLF
K. YOSHIDA

(1906-1982)

\section{SUPPORTING INSTITUTIONS}

UNIVERSITY OF ARIZONA

UNIVERSITY OF BRITISH COLUMBIA

CALIFORNIA INSTITUTE OF TECHNOLOGY

UNIVERSITY OF CALIFORNIA

MONTANA STATE UNIVERSITY

UNIVERSITY OF NEVADA, RENO

NEW MEXICO STATE UNIVERSITY

OREGON STATE UNIVERSITY

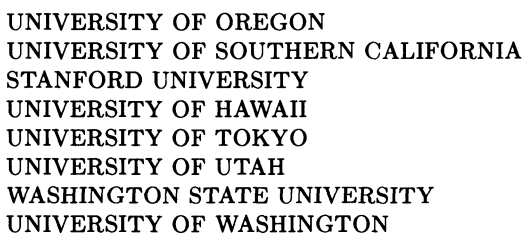

The Supporting Institutions listed above contribute to the cost of publication of this Journal, but they are not owners or publishers and have no responsibility for its content or policies.

\footnotetext{
Mathematical papers intended for publication in the Pacific Journal of Mathematics should be in typed form or offset-reproduced (not dittoed), double spaced with large margins. Please do not use built up fractions in the text of the manuscript. However, you may use them in the displayed equations. Underline Greek letters in red, German in green, and script in blue. The first paragraph must be capable of being used separately as a synopsis of the entire paper. In particular it should contain no bibliographic references. Please propose a heading for the odd numbered pages of less than 35 characters. Manuscripts, in triplicate, may be sent to any one of the editors. Please classify according to the scheme of Math. Reviews, Index to Vol. 39. Supply name and address of author to whom proofs should be sent. All other communications should be addressed to the managing editor, or Elaine Barth, University of California, Los Angeles, California 90024.

There are page-charges associated with articles appearing in the Pacific Journal of Mathematics. These charges are expected to be paid by the author's University, Government Agency or Company. If the author or authors do not have access to such Institutional support these charges are waived. Single authors will receive 50 free reprints; joint authors will receive a total of 100 free reprints. Additional copies may be obtained at cost in multiples of 50 .
}

The Pacific Journal of Mathematics is issued monthly as of January 1966. Regular subscription rate: $\$ 190.00$ a year (5 Vols., 10 issues). Special rate: $\$ 95.00$ a year to individual members of supporting institutions.

Subscriptions, orders for numbers issued in the last three calendar years, and changes of address should be sent to Pacific Journal of Mathematics, P.O. Box 969, Carmel Valley, CA 93924, U.S.A. Old back numbers obtainable from Kraus Periodicals Co., Route 100, Millwood, NY 10546.

The Pacific Journal of Mathematics at P.O. Box 969, Carmel Valley, CA 93924 (ISSN 0030-8730) publishes 5 volumes per year. Application to mail at Second-class postage rates is pending at Carmel Valley, California, and additional mailing offices. Postmaster: send address changes to Pacific Journal of Mathematics, P.O. Box 969, Carmel Valley, CA 93924.

PUBLISHED BY PACIFIC JOURNAL OF MATHEMATICS, A NON-PROFIT CORPORATION Copyright (C) 1989 by Pacific Journal of Mathematics 


\section{Pacific Journal of Mathematics}

\section{Vol. 136, No. 2 December, 1989}

A. K. Agarwal and David Bressoud, Lattice paths and multiple basic hypergeometric series ................................. 209

Adalberto Panobianco Bergamasco and Hermano de Souza Ribeiro, Uniqueness in a doubly characteristic Cauchy problem ........... 229

Thomas Curtis Craven and George Leslie Csordas, Jensen polynomials

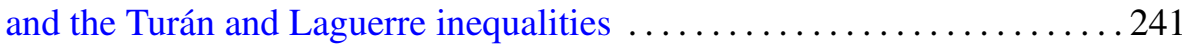

Gary R. Jensen and Marco Rigoli, Harmonic Gauss maps ............ 261

L. G. Kovács and Cheryl Elisabeth Praeger, Finite permutation groups with large abelian quotients $\ldots \ldots \ldots \ldots \ldots \ldots \ldots \ldots \ldots \ldots \ldots \ldots . \ldots \ldots$

Ken-ichi Maruyama, Localization of a certain subgroup of self-homotopy

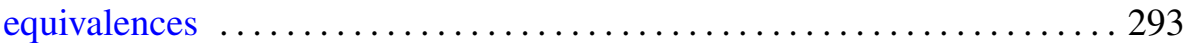

Tomasz Mazur, Canonical isometry on weighted Bergman spaces ......... 303

Bernt Karsten Oksendal, A stochastic Fatou theorem for quasiregular

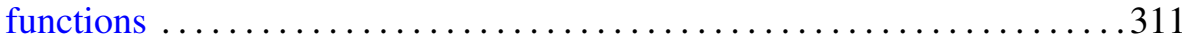

Ian Fraser Putnam, The $C^{*}$-algebras associated with minimal homeomorphisms of the Cantor set ........................ 329

Tom Joseph Taylor, Some aspects of differential geometry associated with

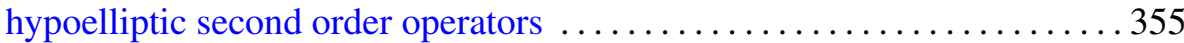

Tom Joseph Taylor, Off diagonal asymptotics of hypoelliptic diffusion equations and singular Riemannian geometry 